\title{
Perturbed hedgehogs: continuous deformation of point defects in biaxial nematic liquid crystals
}

\author{
David Chillingworth \\ Mathematical Sciences \\ University of Southampton \\ Southampton SO17 1BJ UK
}

November 18, 2015

\section{Introduction}

The term liquid crystal describes a fluid in which geometrical structure of individual molecules can induce a tendency to align in certain ways with other molecules or with external magnetic or electric fields or with container boundaries. The degree and nature of molecular alignment strongly influences the optical properties of the liquid crystal, a phenomenon that lies at the heart of the currently ubiquitous liquid crystal display technology. For an overview of liquid crystals see $[8],[9],[10],[27]$ or for more technical theoretical and mathematical background see [7],[24],[25] as well as the excellent survey [20].

An equilibrium state of a body of liquid crystal is called a phase, and the phase transitions (changes from one phase to another) are of particular importance in applications. Some typical phases for rod-like molecules are illustrated in Figure 1. In the first example the nematic phase is determined purely by the overall alignment direction $\mathbf{n}$ (we do not distinguish $\mathbf{n}$ from $-\mathbf{n})$, while in the the smectic and cholesteric phases there is periodic spatial pattern as well.

It is easy to see that for topological reasons there may have to be discontinuities in alignment even in the nematic phase since, for example, a 


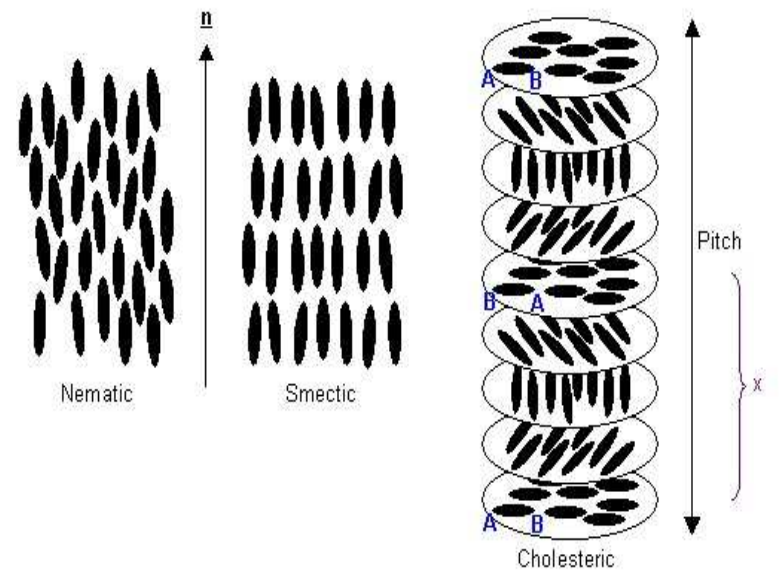

Figure 1: Various liquid crystal phases (Image: University of Cambridge DoITPoMS).

radially-aligned configuration of molecules on the boundary of a disc (in 2D) or ball (in 3D) cannot be extended continuously to fill the interior (for real projective space $R P^{n}$ the homotopy group $\pi_{n}\left(R P^{n}\right)$ is nontrivial, here for $n=1,2)$. The simplest way to attempt to extend over a disc is to maintain the radial alignment arbitrarily closely to the centre of the disc, but then leave the alignment undetermined (isotropic melt) at the centre. Such a configuration may not be physically stable, of course. In $2 \mathrm{D}$, viewing this in polarised light would yield a decomposition of the disc into two bright segments and two dark segments, while more complicated configurations around a singular point yield decompositions with $2 n$ bright and $2 n$ dark segments for $n=1,2, \ldots$ leading to intricate overall patterns of light and dark (Schlieren textures) as shown in Figure 2. An insightful and detailed analysis of these optical phenomena can be found in [25].

\section{Order parameters: the Q-tensor model}

In a small volume of liquid crystal (though much larger than individual molecules) an ensemble of rod-like molecules will align in many different directions, some more likely than others. This tendency can be represented by a probability distribution $p$ on the space of possible directions, that is to say on the unit sphere $S^{2} \subset \mathbf{R}^{3}$, with the understanding that $p(s)=p(-s)$ for $s \in S$ (the rod has no preferred head or tail). The symmetry implies that the mean of $p$ is zero, so the lowest-order meaningful approximation to $p$ is its second moment. This is a quadratic form represented by a $3 \times 3$ real sym- 


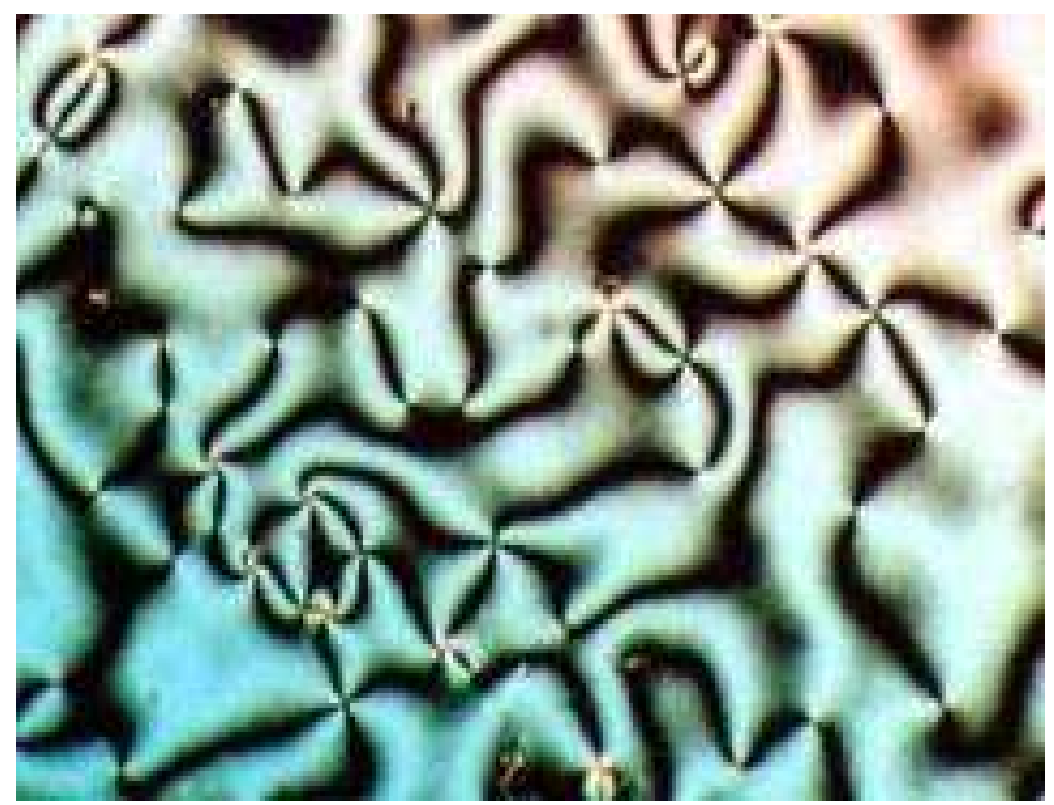

Figure 2: Schlieren texture (Image: Wikipedia).

metric matrix once a coordinate frame in $\mathbf{R}^{3}$ is chosen. The isotropic state, represented by uniform distribution $p$, corresponds to a scalar multiple of the identity matrix. Since we are interested in deviation from isotropy we subtract off this redundant contribution so adjust the matrix to have zero trace. As far as the relevant properties of interaction are concerned, the propensities for molecular alignments are therefore approximately represented at each point $\mathbf{x}$ of the fluid by an element $\mathbf{Q}=\mathbf{Q}(\mathbf{x})$ of the linear space

$$
V=\{3 \times 3 \text { real symmetric traceless matrices }\} \cong \mathbf{R}^{5}
$$

of so-called Q-tensors, with isotropy now corresponding to $\mathbf{Q}=0 \in V$.

\subsection{Uniaxiality and biaxiality}

If $\mathbf{n}$ is a unit vector in $\mathbf{R}^{3}$ and $I$ is the $3 \times 3$ identity matrix then the matrix

$$
\mathbf{Q}=\mathbf{Q}_{\mathbf{n}}:=\mathbf{n n}^{T}-\frac{1}{3} I
$$

has trace zero and has

$$
\begin{aligned}
& \text { eigenvalue } \lambda=\frac{2}{3} \text { with eigenvector } \mathbf{n} \\
& \text { eigenvalue } \lambda=-\frac{1}{3} \text { with } 2 \text {-dim eigenspace } \mathbf{n}^{\perp}
\end{aligned}
$$

and we note that $\mathbf{Q}_{\mathbf{n}}=\mathbf{Q}_{-\mathbf{n}}$. In particular if $\mathbf{n}=( \pm 1,0,0)$ then $\mathbf{Q}_{\mathbf{n}}=$ $\frac{1}{3} \operatorname{diag}(2,-1,-1)$ and likewise for the other axis unit vectors. Positive scalar 


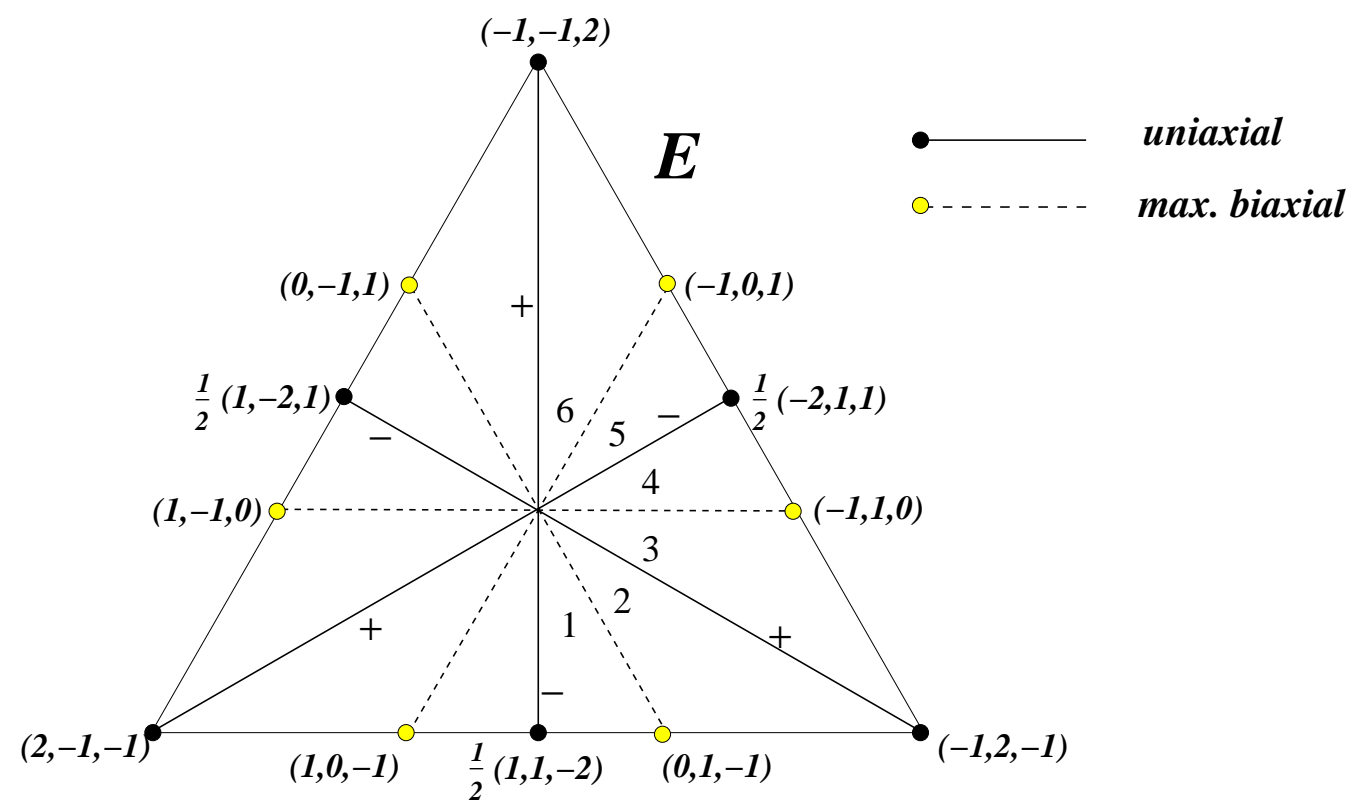

Figure 3: Uniaxial (positive and negative, indicated by signs) and maximally biaxial diagonal (dashed lines) matrices with zero trace. See Section 3.2 for explanation of labels 1-6 in complementary triangular regions.

multiples of any $\mathbf{Q}_{\mathbf{n}} \in V$ are called (positive) uniaxial, while negative scalar multiples are negative uniaxial. We call $\mathbf{n}($ or $-\mathbf{n})$ the principal eigenvector of $\mathbf{Q}_{\mathbf{n}}$. A uniaxial matrix thus represents a tendency of molecules to align locally along the direction of its principal eigenvector, while a negative uniaxial matrix represents a tendency to align in any direction perpendicular to this with equal likelihood.

These are the only cases where $\mathbf{Q} \in V$ has a pair of equal eigenvalues; all other (nonzero) $\mathbf{Q} \in V$ are called biaxial. A biaxial matrix $\mathbf{Q}$ with eigenvalues $c<b<a$ represents molecules with a local propensity to align in the direction of the eigenvector $\mathbf{n}$ with eigenvalue $a$, although with deviation from $\mathbf{n}$ not uniform in all directions orthogonal to $\mathbf{n}$ but dominated by the direction of the eigenvector $\mathbf{m}$ with eigenvalue $b$. See [20] for further careful discussion of the interpretation of the matrix $\mathbf{Q}$.

Clearly some matrices are 'more biaxial' than others, measured by the extent to which their eigenvalues fail to coincide. In this sense the maximally biaxial matrices are those with eigenvalues $\{-1,0,1\}$. The relative configuration of uniaxial and maximally biaxial diagonal matrices is shown in the equilateral triangle $E$ (boundary and interior) of Figure 3, where we write $(a, b, c)$ to denote the matrix $\operatorname{diag}(a, b, c)$. 


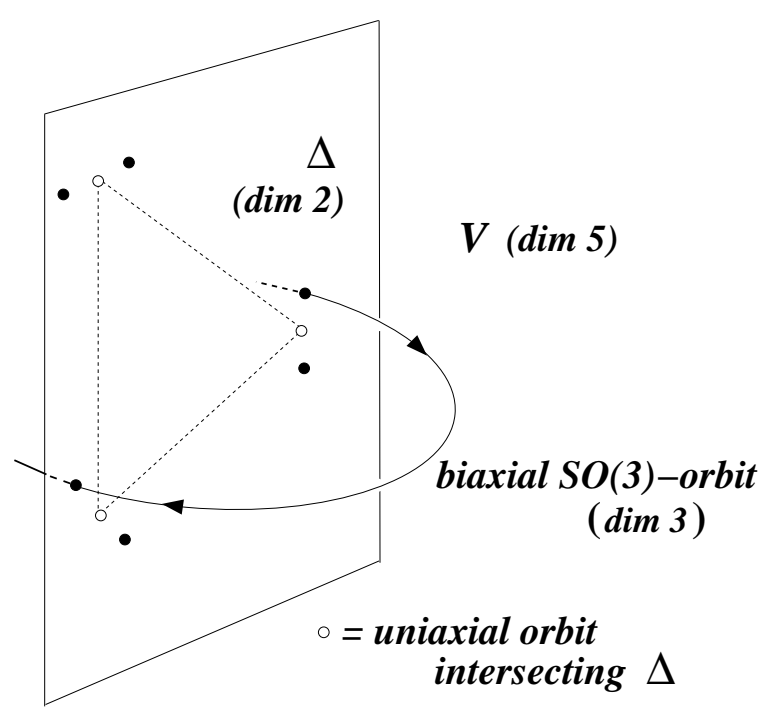

Figure 4: $S O(3)$-orbit in the 5-dimensional space $V$.

\subsection{Action of the rotation group $S O(3)$}

The choice of a matrix to represent the molecular alignment properties depends on a choice of coordinate frame in $\mathbf{R}^{3}$, while the physical properties of the liquid crystal are of course frame-independent. Therefore in studying phase transitions we are interested in properties of $\mathbf{Q}$ that are unchanged by rigid rotations of $\mathbf{R}^{3}$.

The rotation group $S O(3)$ acts on $V$ by conjugation:

$$
R \in S O(3): \quad \mathbf{Q} \mapsto R \mathbf{Q} R^{-1}=R \mathbf{Q} R^{T} .
$$

The familiar fact that every symmetric matrix can be diagonalised by an orthogonal matrix translates to the geometric statement that every $S O(3)$-orbit in $V$ intersects the 2-dimensional subspace $\Delta \subset V$ consisting of diagonal matrices with zero trace. Orbits of biaxial matrices are manifolds of dimension 3 (the group $S O(3)$ has dimension 3$)$ and they intersect $\Delta(\operatorname{dim} 2)$ in isolated points - as we would expect, since $\operatorname{dim} V=2+3=5$. Orbits of uniaxial matrices (positive or negative) have dimension 2 (geometrically they are copies of $R P^{2}$ ) and these also intersect $\Delta$ in isolated points - as we might not expect. The subgroup $\Sigma$ of $S O(3)$ that takes $\Delta$ into itself is isomorphic to the symmetry group $D_{3}$ of an equilateral triangle. Indeed, each uniaxial orbit intersects $\Delta$ in the vertices of an equilateral triangle with symmetry group $\Sigma$, while each biaxial orbit intersects $\Delta$ in six points forming a $D_{3}$-orbit in $\Delta$. An attempt to represent this geometry is given in Figure 4. 


\subsection{Equilibrium states and free energy}

Stability of liquid crystal phases is customarily characterised in terms of minimisation of a free energy function(al) which incorporates terms arising from molecular interactions, material distortions, electromagnetic fields and surface constraints: see [20],[25] for example. In the absence of external fields and away from any boundary or surface the free energy of a configuration $\mathbf{Q}(\mathbf{x})$ in a region $\Omega$ of fluid at temperature $T$ has the form

$$
\mathcal{F}(\mathbf{Q}, T)=\int_{\Omega} F(\mathbf{Q}(\mathbf{x}), \nabla \mathbf{Q}(\mathbf{x}), T) d \mathbf{x}
$$

where typically the frame-independent free energy density function $F$ is the sum $F=F_{B}+F_{E}$ of a bulk term

$$
F_{B}(\mathbf{Q})=\frac{1}{2} a(T) \operatorname{tr} \mathbf{Q}^{2}-\frac{1}{3} b \operatorname{tr} \mathbf{Q}^{3}+\frac{1}{4} c \operatorname{tr} \mathbf{Q}^{4}
$$

and an elastic term (we here take the simplest version)

$$
F_{E}(\nabla \mathbf{Q})=\frac{1}{2} L|\nabla \mathbf{Q}|^{2}
$$

with $b, c, L>0$ and $\operatorname{tr}=$ trace. Stable equilibrium states correspond to global (absolute) minima of the free energy. Note that the isotropic state $\mathbf{Q}=0$ is a global minimum for sufficiently large $a(T)>0$, but becomes a local maximum (unstable) as $a(T)$ decreases through zero. The function $a(T)$ is typically an increasing function of $\left(T-T_{0}\right)$ for some critical temperature $T_{0}$ where $a\left(T_{0}\right)=0$.

It is easy to verify that $F_{B}$ and $F_{E}$ are invariant under the $S O(3)$ action (1) on $V$. There is much literature devoted to studying aspects of the critical point structure of $F_{B}$ even without considering $F_{E}$ : see for example [1],[5] for results, discussion and many further references.

\section{$3 \quad$ Hedgehogs (and other singularities)}

Although models for liquid crystal phase transitions typically assume that the matrix $\mathbf{Q}=\mathbf{Q}(\mathbf{x})$ varies continuously or smoothly with $\mathbf{x} \in \mathbf{R}^{3}$, its eigenbasis may not do so as at uniaxiality or isotropy the eigenbasis is not unique, and this can give rise to local confusion of the phase. For example, the spatially-varying family of $\mathbf{Q}$-tensors given by

$$
\mathbf{Q}(\mathbf{x})=\alpha(r) \mathbf{Q}_{\mathbf{x} / r}
$$




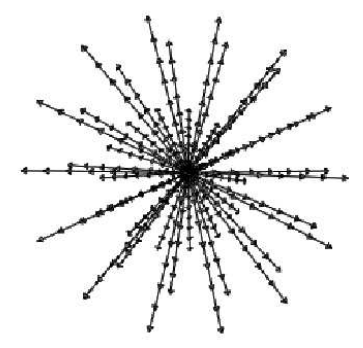

Figure 5: Hedgehog configuration (Image: Pereira, E. et al. Phys.Rev. E87 (2013) 022506).

where $\alpha$ is a suitably regular function of $r=|\mathbf{x}|$ satisfying $\alpha(0)=0$ and $\alpha(r)>0$ for $r>0$ represents a configuration of $\mathbf{Q}$-tensors vanishing at the origin but otherwise uniaxial with principal eigenvector directed radially from the origin in $\mathbf{R}^{3}$ : see Figure 5. This is often called a (radial) hedgehog configuration. In order for it to represent a physically plausible state the scalar factor $\alpha(r)$ has to satisfy a certain ordinary differential equation dictated by the physics. Typically this arises from the Euler-Lagrange equation characterising stationary points of the free energy: see [11, Eqn.(3)] or [12],[13],[17].

The hedgehog configuration represents an equilibrium state for appropriate choice of $\alpha(r)$, but as temperature decreases it becomes unstable in the energetic sense of Section 2.3. Stability of a hedgehog and the nature of the configurations into which a hedgehog relaxes as it loses stability has been much studied in the literature both analytically and numerically: see for example [11],[12],[13],[14],[15],[16],[17],[19],[21],[22]. However, we shall here completely set aside the physical and analytical considerations and focus on the topological constraints that govern the perturbations of a hedgehog, pertinent in this context in view of the results of [18] confirming that energy-minimising configurations $\mathbf{Q}(\mathbf{x})$ are continuous (indeed analytic) in the spatial variable $\mathbf{x}$. Algebraic topological methods have been effectively used in [2],[3] to show that certain uniaxial configurations at the boundary of a fluid region must entail biaxiality elsewhere. The aim of the present paper is to take these ideas one step further in order to predict the likely geometry of uniaxiality and biaxiality that ensues when a hedgehog is continuously perturbed to avoid points of isotropic melt, and to understand in 
particular why typical configurations such as the biaxial torus (or associated ring-disclination) noted in the literature [11],[14],[15],[19],[23] should make an appearance.

\subsection{Geometry close to a hedgehog}

We are interested in perturbing the hedgehog in a neighbourhood of the origin in $\mathbf{R}^{3}$ and so we rescale coordinates if necessary in order to fix this neighbourhood to be the unit ball $B$ with centre the origin. The map

$$
\phi: B \subset \mathbf{R}^{3} \rightarrow V
$$

given by

$$
\phi(\mathbf{x})=\frac{3}{\alpha(1)} \mathbf{Q}(\mathbf{x})=3 \frac{\alpha(r)}{\alpha(1)} \mathbf{Q}_{\mathbf{x} / r}
$$

represents a rescaling of the hedgehog (2) such that if $\mathbf{n}=( \pm 1,0,0)$ then

$$
\phi(\mathbf{n})=3 \mathbf{Q}_{\mathbf{n}}=\operatorname{diag}(2,-1,-1)=: \mathbf{u}(x)
$$

and likewise $\mathbf{u}(y)$ and $\mathbf{u}(z)$ for the other two axes; the purpose of the factor 3 in (3) is simply to avoid carrying a factor $\frac{1}{3}$ in the definition (4). Recall that positive scalar multiples of the matrix $\mathbf{u}(x)$ represent local tendency for molecular alignment along and with rotational symmetry about the $x$-axis, and similarly for $\mathbf{u}(y), \mathbf{u}(z)$.

In describing perturbations of the map $\phi: B \rightarrow V$ we exploit the equivariance (covariance) of $\phi$ with respect to the action of $S O(3)$ on $B$ and on $V$. Specifically, we view the hedgehog as a configuration on the unit disc $D$ in the $x, y$-plane $(z=0)$ as in Figure 6 that is then rotated in $\mathbf{R}^{3}$ about the $y$-axis to give a configuration on $B$, and from this we can build a geometrical picture of the structure of the map $\phi: B \rightarrow V$.

First observe that $\phi$ takes the radius from the origin to $(0,1,0)$ in $D$ to the ray from the origin to $\mathbf{u}(y)$ in $V$. Next, the quarter-circle on the boundary $S$ of $D$ traced out by $(0,1,0)$ under rotation through $-\frac{\pi}{2}$ about the $z$-axis (so clockwise in Figure 6) is taken by $\phi$ to a semicircle from $\mathbf{u}(y)$ to $\mathbf{u}(x)$ in $V$ corresponding to rotation (at twice the speed) about the $\mathbf{u}(z)$-axis in $V$, while the radius from the origin to $(1,0,0)$ in $D$ is taken to the ray from the origin to $\mathbf{u}(x)$ in $V$. The quarter-circle of $S$ in the fourth quadrant is likewise taken by $\phi$ to a semicircle from $\mathbf{u}(x)$ to $\mathbf{u}(y)$ in $V$, and the radius from the origin to $(0,-1,0)$ in $D$ is taken again to the ray from the origin to $\mathbf{u}(y)$ in $V$. Thus the half-disc $D_{+}$of $D$ with $x \geq 0$ is taken by $\phi$ to a cone $K$ in $V$ with vertex at the origin, with axis in the $\mathbf{u}(z)$-direction, and with base 


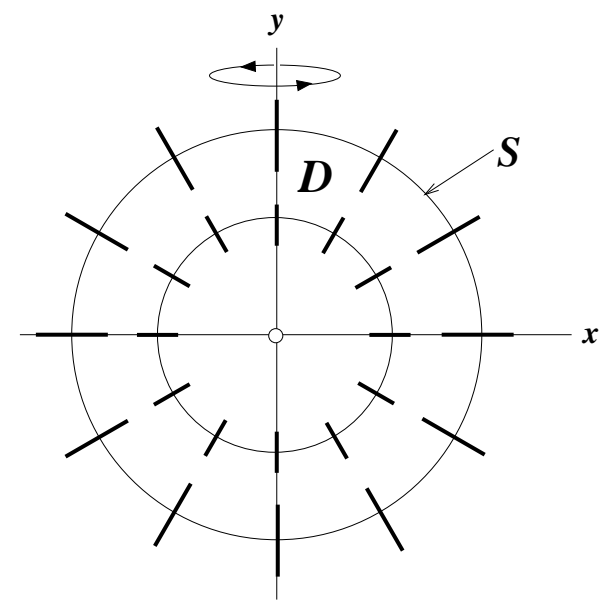

Figure 6: The hedgehog as a rotated configuration on the unit disc in the $x, y$-plane.

circle $C$ passing through $\mathbf{u}(x)$ and $\mathbf{u}(y)$ : see Figure 7 (colour-coded in the electronic version). The half-disc $D_{-}$of $D$ with $x \leq 0$ is taken again to the same cone $K$, so that $\phi \mid D$ is a double cover of $K$ branched at the origin.

Finally, to define $\phi$ on the unit ball $B$ we rotate $D$ about the $y$-axis through angles $\theta$ with $0 \leq \theta \leq \pi$, and observe that the image under $\phi$ of the $\theta$-rotated disc $D_{\theta}$ is obtained by rotating the cone $K$ through angle $2 \theta$ about the $\mathbf{u}(y)$-axis in $V$ : a little harder to visualise now in five dimensions. The restriction of $\phi$ to the boundary sphere of $B$ is a double cover of the projective plane $P$ in $V$ that is the $S O(3)$-orbit of $\mathbf{u}(x)$ (and of $\mathbf{u}(y), \mathbf{u}(z)$ ), while $\phi(B)$ is the positive cone from the origin to $P$.

\subsection{Perturbation of the map $\phi$}

We next ask: What happens to the image of $B$ under typical $C^{1}$ perturbations of the map $\phi$, assuming that $\phi$ is essentially unchanged on the boundary of $B$ ? Here we leave aside the issue of minimising the free energy $\mathcal{F}$, and consider this question purely from a topological point of view in order to gain insight into the geometry that constrains the physics. We list the topologically stable (robust to further small $C^{1}$ perturbations) possibilities that arise under certain simplifying assumptions, while recognising that it is energy minimisation that determines which possibilities could actually occur as physically stable phases in any given case.

Of course, the word 'typical' is here not defined, and indeed there are infinitely many distinct ways in which to perturb the map $\phi$ to give essentially 


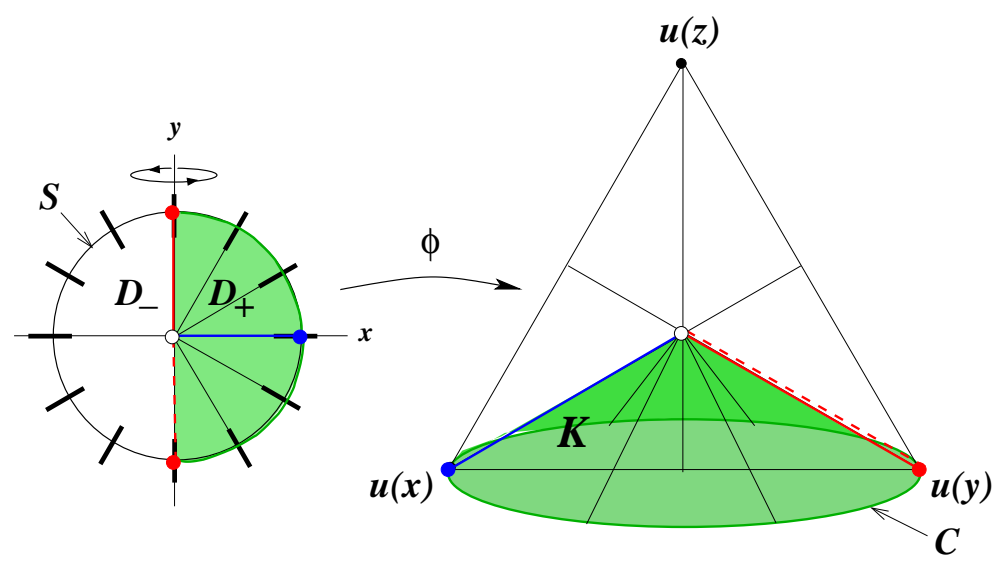

Figure 7: The map $\phi$ takes the half-disc $D_{+}$to a cone $K$ in $V$.

(topologically) distinct configurations of molecular alignments. In order to gain a better hold on the problem we therefore make the following rotational symmetry assumption:

(RS) Rotational Symmetry assumption:The topological type of the configurations of uniaxial and maximally biaxial matrices on the rotated disc $D_{\theta}$ determined by the restriction $\phi_{\varepsilon} \mid D_{\theta}$ is independent of $\theta$.

This assumption means that it suffices to study the geometry of the map $\phi_{\varepsilon} \mid D_{+}$, and in particular how it relates to the uniaxial and maximally biaxial matrices in $V$.

Let $\mathcal{U}, \mathcal{M}$ denote the subsets of $V$ consisting respectively of uniaxial and maximally biaxial matrices with diagonalisations lying in the equilateral triangle $E$ of Figure 3. These sets can be visualised as follows. First consider the result of rotating Figure 3 about the $\mathbf{u}(z)$-axis, as in Figure 8: compare Figure 3 in [3] or Figure 1 in [2]. The uniaxial matrices are now represented by a (double) cone together with its axis, with vertex at the origin excluded. Likewise the maximally biaxial matrices in Figure 8 are represented by a cone coaxial with the uniaxial cone as well as by a disc with centre the origin and orthogonal to the $\mathbf{u}(z)$-axis (origin excluded). The full sets $\mathcal{U}$ and $\mathcal{M}$ are then obtained by rotating these structures through $2 \pi$ about the $\mathbf{u}(y)$-axis in $V$.

The origin has codimension 5 in $V$ and so we expect the image of $\phi_{\varepsilon}$ to avoid points of isotropy. However, each of the subsets $\mathcal{U}$ and $\mathcal{M}$ has codimension 1 in $V$ and so we expect the image of $\phi_{\varepsilon}$ to intersect either or both of them: the geometry will show that it must intersect both. The fact that avoidance of isotropy leads to locations of maximal biaxiality has been 
frequently noted in the literature: see $[2],[4],[6]$.

Let us write

$$
\psi:=\phi \mid D: D \rightarrow V
$$

and likewise $\psi_{\varepsilon}=\phi_{\varepsilon} \mid D$. There are two 'obvious' ways in which $\psi_{\varepsilon}$ can move the cone or tent of Figure 7 to avoid the origin: either downwards (deflation of the tent) so that the perturbed image lies below the original cone (that is, the $\mathbf{u}(z)$ component everywhere decreases except on the 'base' circle $C:=\psi(S)$ ) or upwards (inflation of the tent) so that the perturbed image lies above the original cone (that is, the $\mathbf{u}(z)$ component everywhere increases except on the circle $C$ ). See Figure 9 .

In general the perturbation $\psi_{\varepsilon}$ could also have a small component of rotation about $\mathbf{u}(y)$. However, since $\mathcal{U}$ and $\mathcal{M}$ are preserved by this rotation and it is the intersection of $\psi_{\varepsilon}(D)$ with these sets that concerns us we factor out this rotational component and suppose $\psi_{\varepsilon}(D)$ to lie in the $\mathbf{u}(z)$-rotated triangle $E$ as in Figure 9.

Although Figures 9(a) and (b) capture natural ways to perturb the map $\psi$ it is important to remember that $\psi_{\varepsilon}(D)=\psi_{\varepsilon}\left(D_{+} \cup D_{-}\right)$which is close to the double (branched) cover of the cone $K$ by $\psi=\phi \mid D: D \rightarrow K$ as in Figure 7 . Therefore the geometry of the molecular alignment configuration on $D$ that is associated to $\psi_{\varepsilon}$ may (and does) depend on the location of the branch point $\psi_{\varepsilon}(0)$ relative to $\mathcal{U}$ and $\mathcal{M}$ (to which we can assume it does not belong). To keep track of the possibilities we label the regions of the complement of $E \cap(\mathcal{U} \cup \mathcal{M})$ in $E$ in Figure 3 by the labels 1-6: roughly speaking, regions 1-3 represent deflation while regions $4-6$ represent inflation. Note that the six further regions to the left of the $\mathbf{u}(z)$-axis in Figure 3 are obtained from the six numbered regions by rotation through angle $\pi$ about the $\mathbf{u}(z)$-axis, and thus the corresponding molecular alignment configurations in $D$ are obtained from those of regions 1-6 in $E$ simply by rotating $D$ by $\pi / 2$ about the $z$-axis.

The location of $\psi_{\varepsilon}(0)$ does not itself determine the molecular alignment configuration on $D$ since $\psi_{\varepsilon}(D)$ may still intersect $\mathcal{U} \cup \mathcal{M}$ in a variety of ways. We therefore make a further topological simplicity assumption.

(TS) Topological Simplicity assumption. The topology of the molecular alignment configuration on $D$ determined by the map $\psi_{\varepsilon}: D \rightarrow V$ is as simple as possible given the constraints imposed by the location of $\psi_{\varepsilon}(0)$.

Here topological simplicity means the avoidance of superfluous kinks or bumps in $\psi_{\varepsilon}(D)$ so that the number of connected components of $\psi_{\varepsilon}(D) \cap(\mathcal{U} \cup$ $\mathcal{M})$ is a small as possible. Such variations are controlled by keeping $|\nabla \mathbf{Q}|^{2}$ 


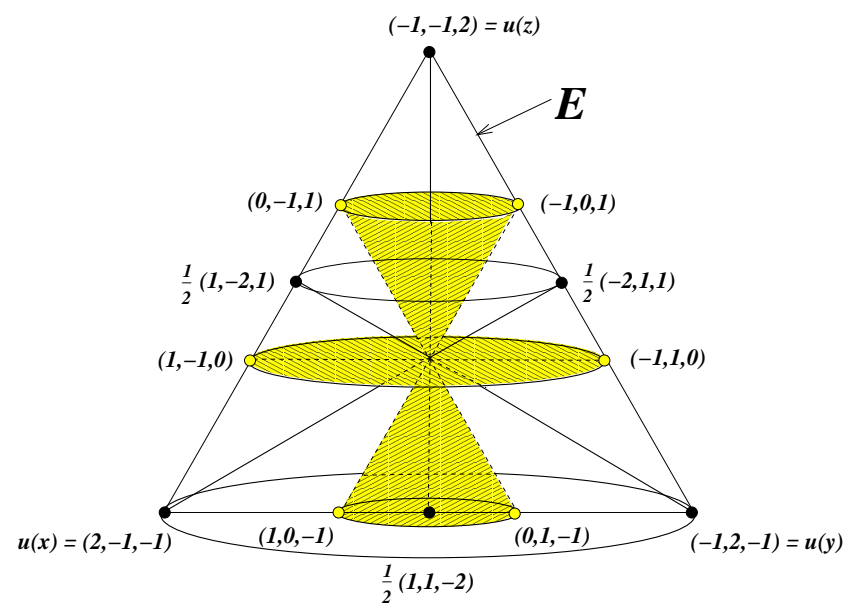

Figure 8: Maximally biaxial diagonal matrices (shown shaded) after rotation of $\Delta$ (diagonal matrices) about the $z$-axis.

as small as is compatible with the topological constraints. This approach seems consistent with minimisation of the free energy, although we offer no analytical proof that (TS) should hold. The intuitive picture is of a soap film no longer pinned at the origin and aiming to be as flat as possible while constrained by the bulk term $F_{B}$ of the free energy.

In the presence of full rotational symmetry about the $y$-axis the inflation/deflation description does not apply so freely, since in particular $\psi_{\varepsilon}$ must then take the $y$-axis in $D$ into the $\mathbf{u}(y)$-axis in $V$ and so the branch point $\psi_{\varepsilon}(0)$ must lie on that axis. The correspondingly constrained versions of Figure 9(a) and (b) are illustrated in Figure 10(a) and (b).

\subsection{The map $\phi_{\varepsilon}$ as a molecular configuration}

The interpretation of $\phi_{\varepsilon}$ as a molecular alignment configuration for the liquid crystal on any subset $W$ of $B$ is governed by the structure of the sets

$$
\mathcal{U}_{W}:=\left\{\mathbf{x} \in W: \phi_{\varepsilon}(\mathbf{x}) \in \mathcal{U}\right\}
$$

and

$$
\mathcal{M}_{W}:=\left\{\mathbf{x} \in W: \phi_{\varepsilon}(\mathbf{x}) \in \mathcal{M}\right\}
$$

which respectively represent the locus in $W$ of the uniaxial and the maximally biaxial matrices. We construct $\mathcal{U}_{B}$ and $\mathcal{M}_{B}$ as follows. First we suppose $\psi_{\varepsilon}$ : $D \rightarrow V$ is transverse (in general position) to $\mathcal{U} \cup \mathcal{M}$ and assuming (TS) we construct $\mathcal{U}_{D}$ and $\mathcal{M}_{D}$ for each of the six choices of location of the point $\psi_{\varepsilon}(0)$. 

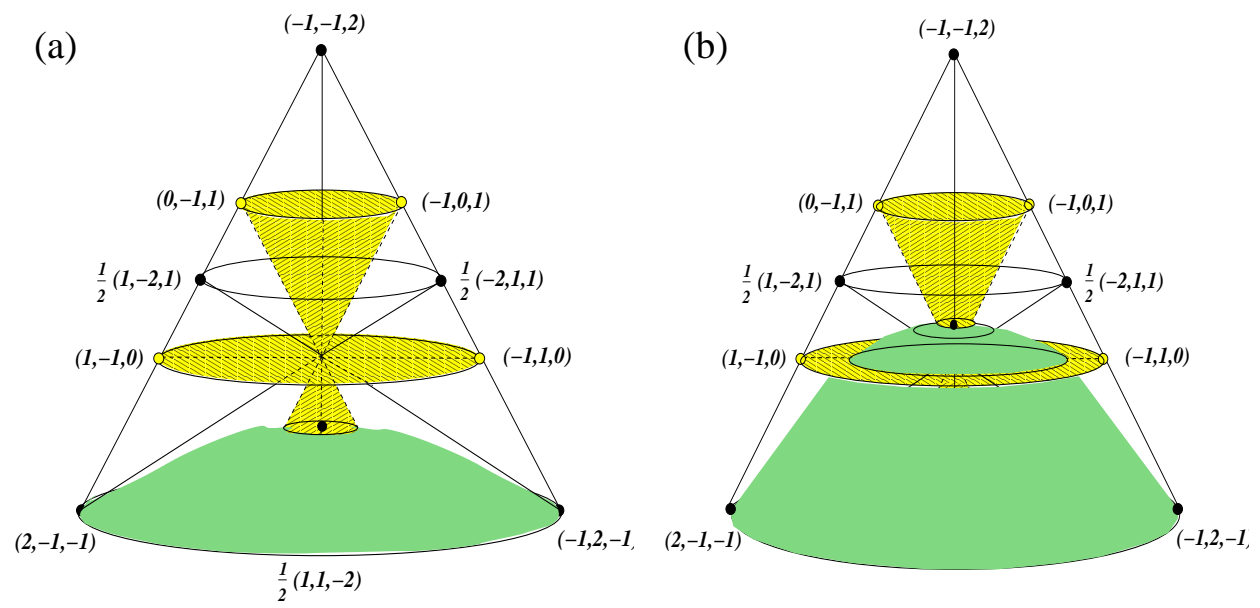

Figure 9: Perturbed map $\psi_{\varepsilon}: D \rightarrow V:$ (a) deflated tent, (b) inflated tent. The image $\psi_{\varepsilon}(D)$ is shaded green while the set of maximally biaxial matrices is shaded yellow. The cone with vertical axis representing uniaxial matrices is left unshaded for transparency.
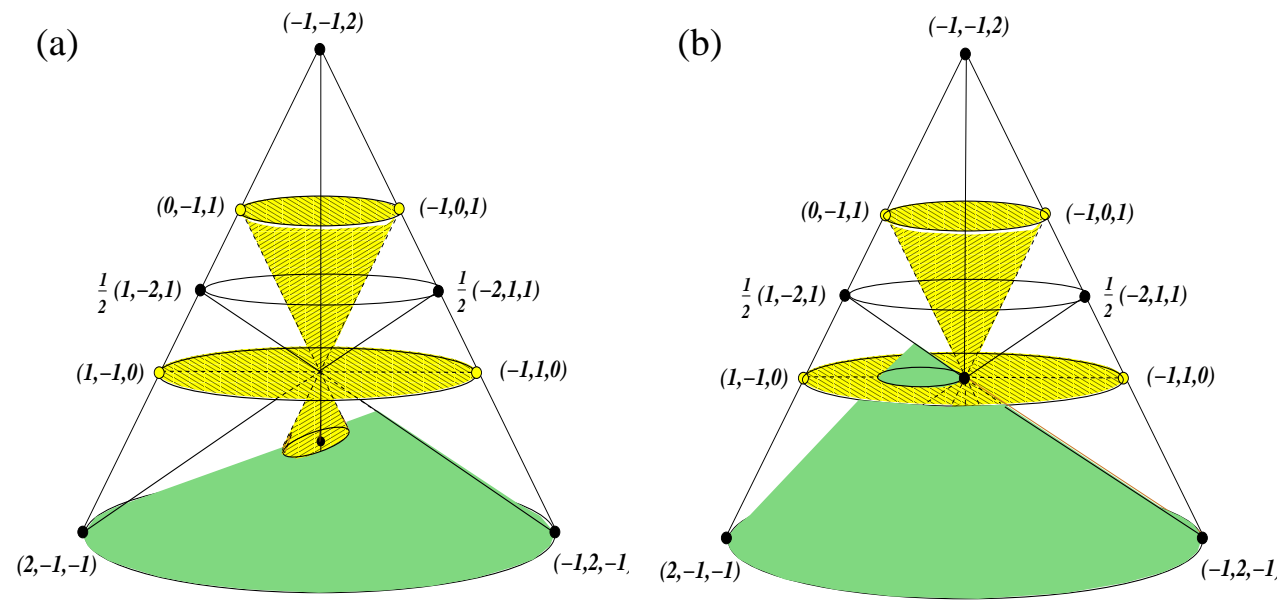

Figure 10: Perturbed map $\psi_{\varepsilon}$ with rotational symmetry about the $y$-axis: (a) deflated tent, (b) inflated tent. Colour coding as in Figure 9. 
We then invoke (RS) to suppose that $\mathcal{U}_{D_{\theta}}$ and $\mathcal{M}_{D_{\theta}}$ vary continuously with $\theta$ and do not change their topological type. If (RS) were not invoked, so that $\phi_{\varepsilon}$ could be far from rotationally symmetric, then the topology of $\mathcal{U}_{D_{\theta}}$ and $\mathcal{M}_{D_{\theta}}$ could vary drastically with $\theta$, and 'typical' configurations would be harder to categorise.

The alert reader will note that for sufficiently small perturbations $\psi_{\varepsilon}$ of the rotationally symmetric maps illustrated in Figure 10 the image $\psi_{\varepsilon}(0)$ of the origin can lie only in regions $2-5$. However, for completeness we include also the slightly larger perturbations with $\psi_{\varepsilon}(0)$ in regions 1 or 6 .

\subsection{Construction of the configuration topology}

As an illustrative example we consider the case when $\psi_{\varepsilon}(0)$ lies in region 1 of Figure 3. Here $\psi_{\varepsilon}$ does not retain rotational symmetry about the $y$-axis, but let us assume temporarily that $\psi_{\varepsilon}$ does retain the symmetry of rotation by $\pi$ about the $z$-axis, so that $\psi_{\varepsilon}\left(D_{+}\right)=\psi_{\varepsilon}\left(D_{-}\right)=\psi_{\varepsilon}(D)$ while $\psi_{\varepsilon}$ takes both the positive and negative $y$-axis in $D$ to the same rib from $\psi_{\varepsilon}(0)$ to $C$ on the deflated tent. We now look at the intersections of $\psi_{\varepsilon}(D)$ with $\mathcal{U}$ and with $\mathcal{M}$.

First observe that $\psi_{\varepsilon}\left(D_{+}\right)$intersects the $\mathbf{u}(z)$-axis of Figure 8 in just one point which lies below $(0,0,0)$ and thus represents a point of negative uniaxiality. Next, $\psi_{\varepsilon}\left(D_{+}\right)$intersects the maximal biaxiality set $\mathcal{M}$ (the lower shaded cone in Figure 8) in a closed loop around that cone: see the diagrammatic Figure 13 where it is indicated by a green broken line in the electronic version. The inverse image of this loop under $\psi_{\varepsilon} \mid D_{+}$is an arc in $D_{+}$with both end points on the $z$-axis and otherwise disjoint from it, and so in this way we obtain Figure 11(1).

The full configuration in the disc $D$ is obtained by adjoining to Figure 11(1) its $\pi$-rotation about the $z$-axis. If we had not assumed $\psi_{\varepsilon}$ to have this symmetry then the configuration in $D$ would nevertheless be topologically equivalent to this.

Applying the Symmetry assumption we then see that the configuration in the 3-dimensional ball $B$ determined by $\phi_{\varepsilon}: B \rightarrow V$ has a (topological) circle of negative uniaxiality points encircling the $y$-axis as well as a sphere of maximal biaxiality points enclosing the origin. There are no points of isotropy, since $\psi_{\varepsilon}(D)$ does not meet the origin in Figure 9 and so $\phi_{\varepsilon}(B)$ does not meet the origin in $V$.

Construction of the configurations in $D_{+}$shown in Figure 11(2)-(6) is carried out in a similar way, with the configurations in $D$ obtained initially by 
applying $\pi$-rotation about the $z$-axis and then possibly a small perturbation preserving the topology, and those in $B$ obtained by applying full rotations about the $y$-axis and then a further possible small perturbation again preserving the topology. The molecular alignment configurations in $B$ in these cases are more complicated to describe. For example, in case 4 in addition to a biaxial torus with uniaxial core there is a biaxial sphere around the origin separated from the torus by a uniaxial cylinder with its two end-circles on the original outer uniaxial sphere, while in case 6 there is a uniaxial ring close to the origin surrounded by spheres that are (moving outwards) biaxial, negative uniaxial, biaxial and uniaxial respectively.

Cases where rotational symmetry about the $y$-axis is to be preserved as illustrated in Figure 10 can be viewed as intermediate cases between those of Figure 11: specifically Figure 10(a) is intermediate between cases 2 and 3 while Figure 10(b) is intermediate between cases 4 and 5 although aligned along the $x$-axis rather than the $y$-axis. The deflation geometry (a) is the easiest to describe, consisting of a (topological) torus of maximal biaxiality with a negative uniaxial core: the familiar biaxial torus. In contrast, he inflation geometry (b) in the symmetric case yields the split core configuration: here there are two points of isotropy on the $y$-axis forming the two poles of a sphere of biaxiality as well as the end-points of an interval of negative $y$ uniaxiality along the $y$-axis. The biaxial torus is well known in the literature: see [14, Fig.1],[15, Fig.8] or [11],[19] where the split core is also introduced. The presence of two points of isotropy in the split core configuration does not contradict the earlier remark that isotropy will typically be avoided for topological reasons because rotationally-symmetric configurations are far from typical and different dimensional calculations apply.

In the wider context these intermediate configurations are highly degenerate, however, because in general at no stage during a transition between two configurations of Figure 11 need the image of the $y$-axis under $\psi_{\varepsilon}$ lie entirely in the set of $y$-uniaxial matrices. A more careful analysis of transition states would require further detailed assumptions on the nature of the perturbation $\psi_{\varepsilon}$.

\section{Conclusion}

We have exhibited several configurations of uniaxial and maximally biaxial matrices that arise from a $C^{1}$ perturbation of a 3-dimensional hedgehog for nematic liquid crystals using the $\mathbf{Q}$-tensor model. These are in some sense typical, in that they correspond to displacements of the hedgehog point away 

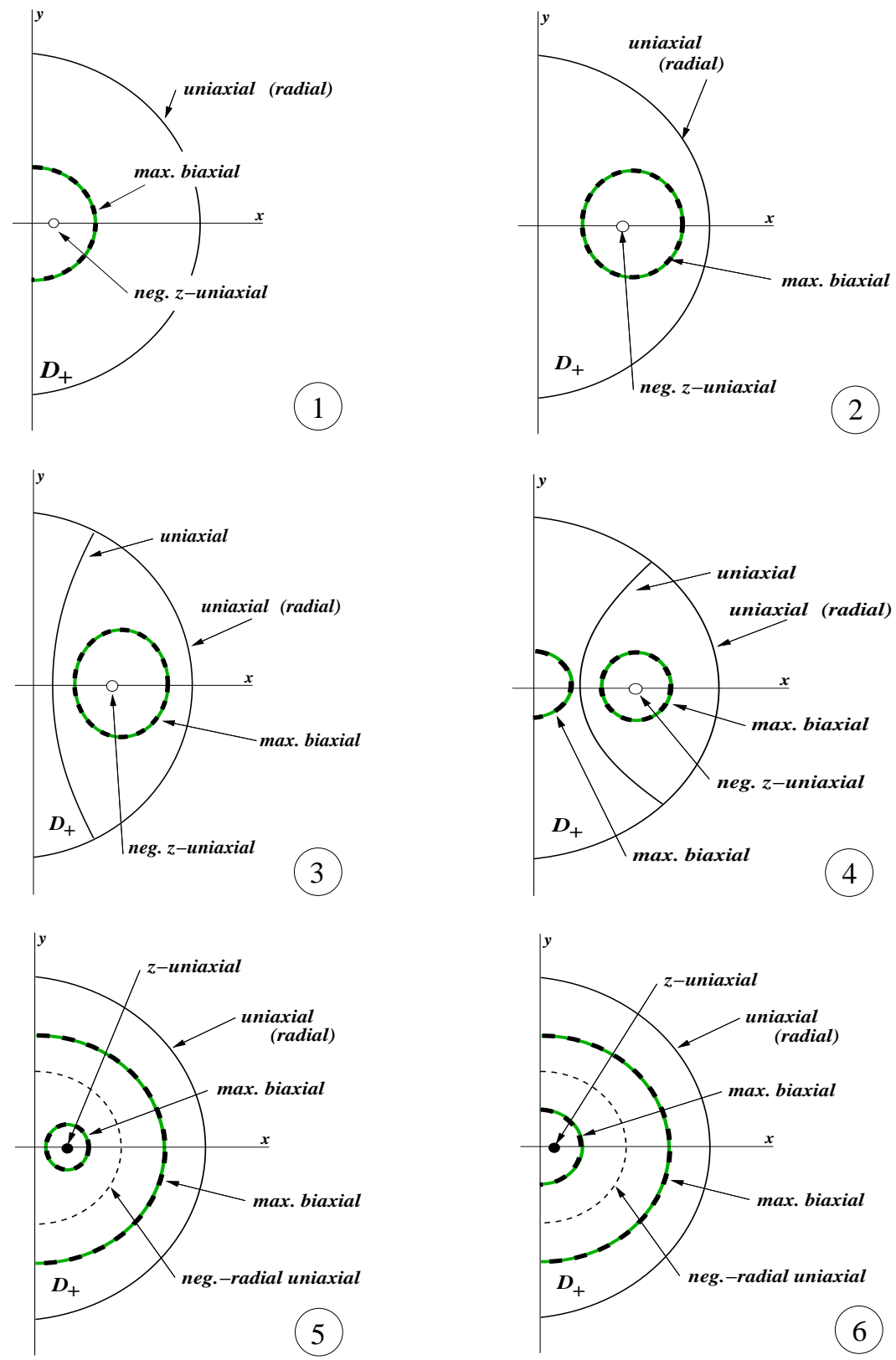

Figure 11: Uniaxial and maximally biaxial configurations in $D_{+}$after perturbations taking the origin to each of the regions 1-6 in Figure 3. 
(a)

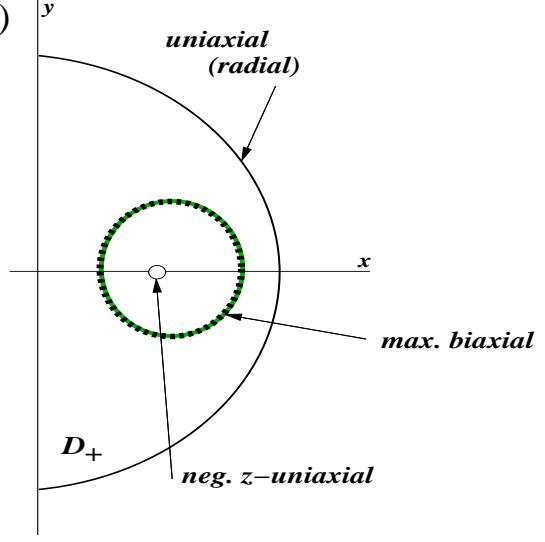

(b)

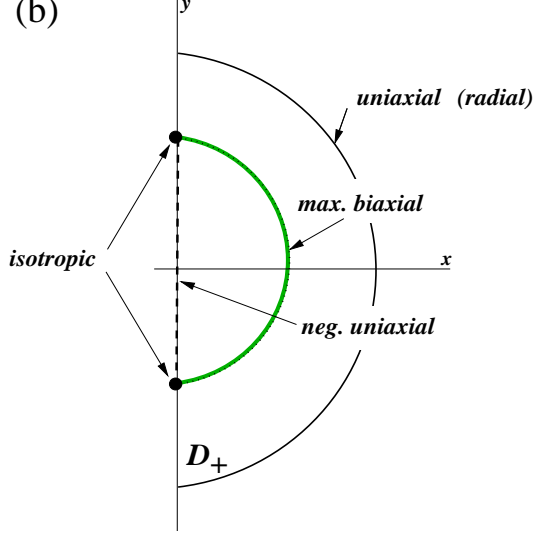

Figure 12: Uniaxial and maximally biaxial configurations in $D_{+}$after perturbations retaining rotational symmetry: (a) deflation, (b) inflation.

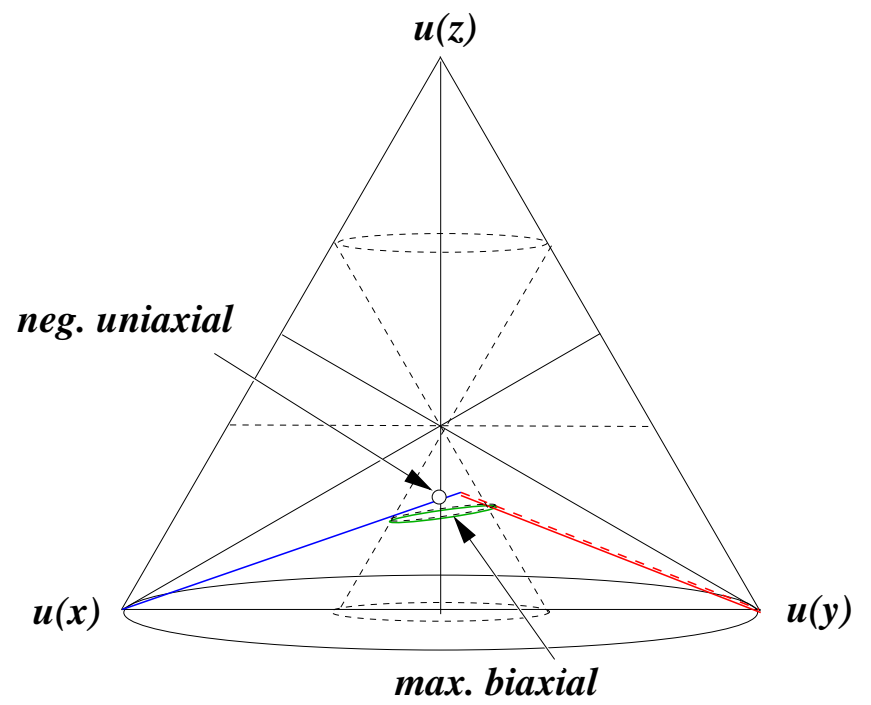

Figure 13: Deflated tent $\psi_{\varepsilon}\left(D_{+}\right)=\psi_{\varepsilon}\left(D_{-}\right)$with vertex in region 1 and with $\psi_{\varepsilon}$ invariant under $\pi$-rotation about $z$-axis. 
from isotropy to neither a uniaxial nor maximally biaxial state and with controlled $C^{1}$ perturbation of the rest of the hedgehog. However, they are based on simplifying assumptions about the topological nature of the perturbation, and can do no more than form a template for a more detailed bifurcation analysis. Under less restrictive assumptions on the geometry of the perturbation $\phi_{\varepsilon}: B \rightarrow V$ other more complicated configurations may arise, although it is prudent to recall again that the nature of the perturbation will in practice be governed by physical considerations (minimisation of free energy) and the exploration of topological possibilities may become less relevant. It is worth noting that, following the soap-film analogy of Section 3.2, the configuration that is closest to rotationally symmetric while at the same time relaxing rather than stretching the soap-film is that corresponding to Figure 11(2), namely the biaxial torus.

Acknowledgements. I am grateful to the anonymous referees for helpful and informative comments that have led to significant improvements in the paper, and to Paolo Biscari, Chuck Gartland and Epifanio Virga for valuable conversations about hedgehogs. I am grateful also for research support from the Isaac Newton Institute, Cambridge, the Leverhulme Trust and the Basque Center for Applied Mathematics (BCAM), Bilbao, Spain.

\section{References}

[1] Allender, D. and Longa, L.(2008), Landau-de Gennes theory of biaxial nematics reexamined, Phys. Rev. E 7811704.

[2] Biscari, P. and Peroli, G. G. (1997), A hierarchy of defects in biaxial nematics. Commun. Math. Phys. 186, 381-392.

[3] Biscari, P., Peroli, G. G. and Sluckin, T. J. (1997), The topological microstructure of defects in nematic liquid crystals, Mol. Cryst. Liq. Cryst 292, 91-101.

[4] Canevari, G. (2015), Biaxiality in the asymptotic analysis in a 2D Landau-de Gennes model for liquid crystals, ESAIM Control Optim. Calc. Var. 21, 101-137.

[5] Chillingworth, D. R. J. (2015), Critical points and symmetries of a free energy function for biaxial nematic liquid crystals, Nonlinearity 28, $1483-1537$. 
[6] Contreras, A. and Lamy, X. (2014), Biaxial escape in nematics at low temperature, http://arXiv.org/abs/1405.2055.

[7] de Gennes P. G. and Prost J. (1993), The Physics of Liquid Crystals 2nd ed. Clarendon Press.

[8] Demus, D., Goodby, J., Gray, G. W., Spiess, H.-W. and Vill, V. (Eds) (1999), Physical Properties of Liquid Crystals, Wiley-VHC.

[9] Dunmur, D. and Sluckin, T. (2010), Soap, Science and Flat-Screen TVs: a History of Liquid Crystals, Oxford University Press.

[10] Fisch, M. R. (2004), Liquid Crystals, Laptops and Life, World Scientific.

[11] Gartland, E. C. and Mkaddem, S. (1999), Instability of radial hedgehog configurations in nematic liquid crystals under Landau-de Gennes freeenergy models, Phys. Rev. E 59 563-567.

[12] Henao, D. and Majumdar, A. (2012), Symmetry of uniaxial global Landau-de Gennes minimizers in the theory of nematic liquid crystals, SIAM J. Math. Anal. 44 3217-3241. Corrigendum: SIAM J. Math. Anal. 45 3872-3874.

[13] Ignat, R., Nguyen, L., Slastikov, V., and Zarnescu, A. (2015), Stability of the melting hedgehog in the Landau-de Gennes theory of nematic liquid crystals, Arch. Rat. Mech. Anal. 215 633-673.

[14] Kralj, S. and Virga, E.G. (2001), Universal fine structure of nematic hedgehogs, J. Phys. A 34 829-838.

[15] Kralj, S., Virga, E. G. and Žumer, S. (1999), Biaxial torus around nematic point defects, Phys. Rev. E 60 1858-1866.

[16] Kralj, S., Žumer, S. and Allender, D. W. (1991), Nematic-isotropic phase transition in a liquid-crystal droplet, Phys. Rev. A 43 2943-2952.

[17] Majumdar, A. (2012), The radial-hedgehog solution in Landaude Gennes theory for nematic liquid crystals, Euro. J. Appl. Math. 23 $61-97$.

[18] Majumdar, A. and Zarnescu, A. (2010), Landau-De Gennes theory of nematic liquid crystals: the Oseen-Frank limit and beyond, Arch. Rat. Mech. Anal. 196, 227-280. 
[19] Mkaddem, S. and Gartland, E. C. (2000), Fine structure of defects in radial nematic droplets, Phys. Rev. E 62 6694-6705.

[20] Mottram, N. J. and Newton, C. P. J. (2014), Introduction to Q-tensor theory, http://arXiv.org/abs/1409.3532.

[21] Penzenstadler, E. and Trebin, H.-R. (1989), Fine structure of point defects and soliton decay in nematic liquid crystals, J. Phys. France 50 1027-1040.

[22] Rosso, R. and Virga, E. G. (1996), Metastable nematic hedgehogs, J. Phys. A 294247.

[23] Sonnet, A., Kilian, A. and Hess, S. (1995), Alignment tensor versus director: Description of defects in nematic liquid crystals, Phys. Rev. E $52718-722$.

[24] Sonnet, A. M. and Virga, E. G. (2012), Dissipative Ordered Fluids, Springer.

[25] Virga, E. G. (1995), Variational theories for liquid crystals, CRC Press.

[26] Virga E. G. (2000), Exotic applications of liquid crystals, in ICIAM 99, J. M. Ball and J. C. R. Hunt Eds., Oxford University Press, 246-258.

[27] Sluckin, T. (2000), The liquid crystal phases: physics and technology, Contemp. Physics 41 37-56. 